\title{
Fatores genéticos e ambientais da atividade física. Um estudo em famílias com três gerações
}

\author{
Physical activity level of three generation families. \\ Genetic and environmental factors
}

\author{
Raquel Nichele de Chaves ${ }^{1}$ \\ Michele Caroline de Souza \\ Daniel Santos \\ André Seabra \\ Rui Garganta \\ José António Ribeiro Maia
}

1 Universidade do Porto. Desporto, Faculdade de Desporto. Laboratório de Cineantropometria e Estatística Aplicada. Porto. Portugal.

Recebido em 15/01/10 Revisado em 04/02/10 Aprovado em 22/03/10

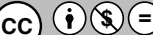

Resumo - Os objetivos do estudo foram: (1) verificar a presença indireta de transmissão vertical de fatores genéticos nos níveis de atividade física (AF) e comportamento sedentário (CS) ao longo de três gerações e (2) estimar a magnitude dos fatores genéticos e ambientais. A amostra foi constituída por 1034 indivíduos de 100 famílias, com três gerações, provenientes da região de Lisboa, Portugal. A avaliação da AF e CS foi realizada por meio do IPAQ, forma curta. Os fenótipos avaliados foram: atividade física total (AFT); vigorosa (AFV); moderada (AFM); caminhada; tempo sentado $\left(T_{S}\right)$, tempo a ver televisão $\left(T_{T V}\right)$ e classificação do nível de AF. Calculou-se o índice de massa corporal (IMC). A estrutura familiar e o comportamento genérico das variáveis entre familiares foram analisados no software Pedstats. A heritabilidade $\left(\mathrm{h}^{2}\right)$ e o efeito do ambiente partilhado foram estimados pelo método de máxima verossimilhança implementado no software Solar. A representação gráfica foi feita no software HLM. Ajustou-se os resultados às covariáveis: sexo, idade, sexo*idade, idade $^{2}$, sexo*idade ${ }^{2}$ e IMC. Foi adotado um nível de significância de 0,05. As estimativas de $h^{2}$ foram as seguintes: AFV h$h^{2}=0,35 \pm 0,06$ ( $\left.p<0.0001\right) ; A F M h^{2}=0,29 \pm 0,06$ $(\mathrm{p}<0.0001)$; caminhada $\mathrm{h}^{2}=0,40 \pm 0,06(\mathrm{p}<0.0001) ;$ AFT $\mathrm{h}^{2}=0,28 \pm 0,06 ; \mathrm{T}_{\mathrm{S}} \mathrm{h}^{2}=0,29 \pm 0,06$ $(\mathrm{p}<0.0001) ; \mathrm{T}_{\mathrm{TV}} \mathrm{h}^{2}=0,15 \pm 0,06(\mathrm{p}<0.003)$ e determinação do nível de $\mathrm{AF} \mathrm{h}^{2}=0,35 \pm 0,14$ $(\mathrm{p}<0.007)$. O efeito ambiental partilhado não foi significativo. Os resultados indicam que os fatores genéticos podem contribuir entre 15 a $40 \%$ da variabilidade total nos diferentes fenótipos da AF e CS, considerada influência baixa a moderada. Fatores ambientais não-transmitidos correspondem à maior contribuição na determinação desses fenótipos.

Palavras-chave: Epidemiologia genética; Atividade física; Heritabilidade.

Abstract - This study aims (1) to investigate the presence of familial aggregation in physical activity (PA) levels and sedentary behavior (SB) among members of three generations families and (2) to estimate the magnitude of additive genetic influences on PA and SB phenotypes. The sample consisted of 100 extended families covering three generations $(n=1034)$, from the Lisbon area, Portugal. Phenotypes were assessed via the short version of the self-administered International Physical Activity Questionnaire (IPAQ-SF). Measured phenotypes: total physical activity (TPA); vigorous (VPA); moderate (MPA); walking; time spent in sitting time (ST), watching television (WT) and PA levels classification. Body mass index (BMI) was calculated. Exploratory family analysis in all phenotypes was conducted in PEDSTATS software. The genetic component $\left(h^{2}\right)$ and shared environmental effect were estimated using maximum likelihood implemented in the SOLAR software package. All graphs were done in HLM software. Sex, age, sex*age, age ${ }^{2}$, sex*age $e^{2}$ and BMI were used as covariates. Significant level was set at 0,05. Genetic component estimates $\left(h^{2}\right)$ were as follows: TPA $h^{2}=0,28 \pm 0,06(p<0.0001)$; VPA $h^{2}=0,35 \pm 0,06(p<0.0001) ; \mathrm{MPA} h^{2}=0,29 \pm 0,06(p<0.0001) ;$ walking $h^{2}=0,40 \pm 0,06(p<0.0001)$; ST $h^{2}=0,29 \pm 0,06(p<0.0001) ; W T h^{2}=0,15 \pm 0,06(p<0.003)$ and determination of the level physical activity $h^{2}=0,35 \pm 0,14(p<0.007)$. Shared environmental effect was not significant. These results showed a low-to-moderate genetic contribution, between $15 \%$ to $40 \%$ of the total variability, in the PA and SB phenotypes. The genetic factors have low to moderate influence in this sample. Non-shared environmental factors appear to have the major contribution in these phenotypes.

Key words: Genetic epidemiology; Physical activity; Heritability. 


\section{INTRODUÇÃO}

Em anos recentes, estudos epidemiológicos têm sugerido uma relação consistente entre estilos de vida fisicamente ativos e estados de saúde ${ }^{1-3}$. As evidências consideram, também, o efeito significativo da prática de atividade física (AF) moderada a elevada na prevenção de inúmeras doenças crônicas, reduzindo riscos e prevalência de morte prematura ocasionada por tais desordens $s^{4-5}$. Decorre daqui a necessidade em entender a magnitude e significados dos fatores que "determinam" os níveis de AF, de modo independente e em interação, de forma a promover estratégias de intervenção e promoção da prática regular desse comportamento ${ }^{6}$.

A compreensão dos determinantes da AF tem progredido na área dos fatores ambientais, mostrando forte tendência a abordagens mais "ecológicas". Estudos prévios reportam, de modo sistemático, a correlação entre diferentes fatores de natureza ambiental e o comportamento da AF em diversos contextos, destacando determinantes potenciais dos níveis de AF como o suporte parental e outros efeitos familiares ${ }^{8-9}$. Embora significativa, a influência familiar sugere muitos questionamentos quanto aos possíveis efeitos genéticos e culturamente transmitidos, além das diferentes exposições a esses efeitos entre as gerações.

No que concerne à magnitude dos fatores genéticos, pesquisas no domínio da Epidemiologia Genética têm apresentado influência genética baixa a moderada na variância total dos diferentes fenótipos da $\mathrm{AF}^{10-14}$, envolvendo, também, o comportamento sedentário (CS) $)^{15-17}$. São resultados amplamente divergentes e, em sua maioria, baseados na transmissão de características entre duas gerações, i.e., entre membros de uma família nuclear. Os dados a partir de um design com famílias nucleares não contêm informação suficiente para estimar de maneira isolada e quantitativa a contribuição relativa dos componentes genéticos e ambientais partilhados, pois avaliam a fração da variância fenotípica atribuível aos fatores combinados de todas as influências familiares ${ }^{18}$. Os fatores ambientais partilhados no seio de cada família, quando não controlados, podem se tornar fatores de confundimento da influência genética na variabilidade dos níveis de AF e sedentarismo. $\mathrm{O}$ delineamento com pedigrees extensos favorece o controle desses efeitos, bem como a diversidade de relações entre diferentes graus de parentesco, estruturando de forma precisa as covariâncias esperadas em um modelo poligênico ${ }^{19}$.
Devido à emergente necessidade de entender a magnitude dos determinantes genéticos e ambientais na variabilidade dos níveis de AF e do CS, à inconsistência dos resultados reportados até a recente data e à escassez de estudos com delineamento baseado em pedigrees extensos, esta pesquisa percorre os propósitos seguintes: (1) verificar a presença indireta de transmissão vertical de fatores genéticos nos níveis de AF e no CS entre membros familiares de três gerações, e (2) estimar a magnitude dos fatores genéticos e ambientais responsáveis pela variabilidade nos níveis de AF e no CS, em termos populacionais.

\section{PROCEDIMENTOS METODOLÓGICOS}

\section{Amostra}

O estudo é de natureza transversal com delineamento baseado em pedigrees extensos. A amostra foi constituída por 1034 indivíduos pertencentes a 100 famílias de três gerações (517 homens e 517 mulheres), provenientes da região de Lisboa, capital de Portugal, apresentando idades entre os 7 e os 85 anos. A amostragem foi efetuada com base no voluntariado das famílias, as quais foram avaliadas após a assinatura do consentimento livre e informado. A dimensão média das famílias foi de 10,34 membros, variando entre 9 e 16 familiares. Devido a dados incompletos, reduziu-se o número de indivíduos analisados, totalizando 738 participantes das 100 famílias envolvidas (Tabela 1).

\section{Níveis de Atividade Física}

Os fenótipos da AF foram avaliados por meio do Questionário Internacional de Atividade Física, forma curta (IPAQ-SF). Esse instrumento tem propriedades de medida aceitáveis no controle dos níveis de AF população com validação internacional previamente reportada e métodos estandardizados. No que concerne à fiabilidade, relatou-se um coeficiente de correlação intraclasse de 0.7-0.8. Para a determinação da validade, esse instrumento foi comparado a dados da acelerometria, reportando, aproximadamente, $r h o=0.30$. IPAQ-SF também é recomendado a pesquisas sobre o controle nacional quanto aos níveis de $\mathrm{AF}^{20-21}$.

A aplicação desse instrumento foi feita após explicação do avaliador sobre o objetivo de cada questão. Os participantes com idades inferiores a 15 anos foram entrevistados por uma equipe previamente treinada a partir de uma abordagem padronizada. Aos outros indivíduos, o questionário foi auto-administrado. Após uma semana, foi 
realizado o reteste com 10 famílias, no sentido de obter estimativas de fiabilidade.

Os fenótipos avaliados pelo IPAQ-SF foram os seguintes: AF vigorosa (AFV), AF moderada (AFM), caminhada e CS, nomeadamente, tempo sentado $\left(T_{S}\right)$ e dispendido a assistir televisão $\left(T_{T V}\right)$. As medidas obtidas referem-se à frequência (dias por semana) e à duração (minutos por dia). De acordo com o protocolo oficial estandardizado ${ }^{22}$, os resultados podem ser apresentados por escalas contínuas do dispêndio energético semanal, expresso pelo equivalente metabólico (MET/minutos/semana). Sendo um MET, do inglês metabolic equivalent, o valor aproximado do $\mathrm{VO}_{2}$ de repouso, os valores de referência para o cálculo do dispêndio energético são: 8.0 METs para AFV; 4.0 METs para AFM; e 3.3 METs para caminhada. A AF total (AFT) é obtida por meio da soma dos valores reportados para AFV, AFM e caminhada. Para o comportamento sedentário, atribui-se o equivalente metabólico em repouso, mantendo os valores em minutos por dia.

Após os cálculos, propõe-se uma classificação categórica dos indivíduos avaliados em três níveis de AF: pouco ativos; moderadamente ativos e muito ativos. A classificação considera os valores reportados em METs, bem como a frequência e a duração da AF. Neste estudo, os indivíduos foram classificados em duas categorias: pouco ativos (categoria 1) e de atividade moderada-a-extenuante (categorias 2 e 3), pois a análise estatística exigia que as variáveis fossem contínuas ou binárias.

Para investigar os parâmetros associados com os níveis de AF, foram medidos; a estatura, massa corporal; o índice de massa corporal (IMC), o efeito do ambiente partilhado é definido como household effects. A estatura foi medida com um antropômetro portátil da marca Siber Hegner $\AA$, com precisão de 0,1 centímetro $(\mathrm{cm})$, de acordo com as referências de Lohman et $\mathrm{a}^{23}$. A massa corporal foi medida com uma balança (Secca) de precisão de 0,1 quilograma $(\mathrm{kg})$. O indivíduo deveria estar na posição antropométrica de referência ${ }^{23}$, descalço e vestindo roupas leves. $\mathrm{O}$ IMC foi calculado por meio da razão entre a massa corporal e a estatura ao quadrado, obtendo um valor final expresso em $\mathrm{kg} / \mathrm{m}^{2}$. Para determinar o efeito do ambiente partilhado, questionou-se cada avaliado sobre as pessoas com quem morava, estabelecendo uma codificação contínua capaz de identificar os sujeitos que conviviam entre si, i.e., na mesma morada.

\section{Análise Estatística}

A análise exploratória dos dados para verificação de possíveis erros da informação e presença de outliers foi realizada no software SPSS versão 17.0, bem como as medidas de fiabilidade, com os cálculos dos coeficientes de correlação intraclasse (R) e os respectivos intervalos de confiança $\left(\mathrm{IC}_{95 \%}\right)$. Devido aos elevados valores de assimetria e achatamento, os fenótipos da AF foram transformados para o inverso da distribuição normal. Para verificar a estrutura de cada família e analisar o comportamento genérico das variáveis entre os diferentes membros da família, utilizou-se o software PEDSTATS. O cálculo da heritabilidade $\left(\mathrm{h}^{2}\right)$ foi realizado no software SOLAR versão 4.0. Destaca-se que $a h^{2}$ e o efeito do ambiente partilhado foram estimados pelo método de máxima verossimilhança implementado nesse software. Inicialmente, foi formulado um modelo designado de esporádio (ou seja, ausência de covariância entre familiares para os distintos fenótipos). O valor de ajustamento desse modelo (Loglikelihood) é contrastado com o que resulta de um modelo poligênico. A diferença entre os dois Loglikelihoods tem uma distribuição aproximada à do qui-quadrado com 1 grau de liberdade. Espera-se um abaixamento de valor de Loglikelihood no modelo poligênico e que o resultado da estatística de qui-quadrado seja significativo. As representações gráficas foram efetuadas no software HLM versão 6.02. Os níveis de AF e CS foram ajustados às covariáveis idade, sexo, idade ${ }^{2}$, sexo*idade, sexo*idade ${ }^{2}$ e IMC. O nível de significância adotado foi de 0,05.

\section{RESULTADOS}

Os resultados deste estudo mostram uma fiabilidade elevada, com todos os valores pontuais de $\mathrm{R}$ acima 0,80 . O intervalo de confiança situou-se entre 0.84 e 1.0, indicando a excelente qualidade dos dados.

A Tabela 1 apresenta a estratificação da amostra por geração e sexo, bem como os valores médios de idade e IMC. Em média, os valores de IMC dos indivíduos da primeira e segunda gerações são similares, situando-os próximos aos níveis de sobrepeso. A terceira geração, ao contrário, apresenta tendência de índices normoponderais.

A informação sobre os níveis de AF e CS é apresentada na Tabela 2. Optou-se pelos valores medianos e de amplitude interquartílica, devido ao fato de a assimetria e o achatamento serem elevados. Verifica-se que metade dos indivíduos da primeira e segunda gerações apresentam gasto energético para AFV igual a 0 . Tal valor repete-se para AFM nos familiares da primeira geração. Os níveis de AFT reduzem ao longo da idade, enquanto, os valores do CS mostram similaridade e certa instabilidade intergeracional. 
Tabela 1. Medidas descritivas da idade e IMC da amostra.

\begin{tabular}{|c|c|c|c|c|c|c|}
\hline \multirow{3}{*}{ Variáveis } & \multicolumn{6}{|c|}{ 1. Geração } \\
\hline & \multicolumn{3}{|c|}{ Avô } & \multicolumn{3}{|c|}{ Avó } \\
\hline & $\mathrm{n}$ & $m \pm d p$ & amplitude & $\mathrm{n}$ & $m \pm d p$ & amplitude \\
\hline Idade (anos) & 104 & $74,4 \pm 10,7$ & $51-85$ & 106 & $76,4 \pm 7,6$ & $52-84$ \\
\hline \multirow[t]{4}{*}{ IMC $\left(\mathrm{kg} / \mathrm{m}^{2}\right)$} & 104 & $25,4 \pm 1,7$ & $22-31,6$ & 106 & $24,3 \pm 2,5$ & $20,5-33,8$ \\
\hline & \multicolumn{6}{|c|}{ 2.. Geração } \\
\hline & \multicolumn{3}{|c|}{ Pai/Tio } & \multicolumn{3}{|c|}{ Mãe/Tia } \\
\hline & $n$ & $m \pm d p$ & amplitude & $\mathrm{n}$ & $m \pm d p$ & amplitude \\
\hline Idade (anos) & 147 & $41,9 \pm 5,2$ & $28-50$ & 175 & $40.9 \pm 4,9$ & $27-50$ \\
\hline \multirow[t]{4}{*}{ IMC $\left(\mathrm{kg} / \mathrm{m}^{2}\right)$} & 147 & $25,7 \pm 1,6$ & $20,8-31,7$ & 175 & $24,5 \pm 1,8$ & $18,4-31,2$ \\
\hline & \multicolumn{6}{|c|}{ 3. a Geração } \\
\hline & \multicolumn{3}{|c|}{ Filho/Neto } & \multicolumn{3}{|c|}{ Filha/Neta } \\
\hline & $\mathrm{n}$ & $m \pm d p$ & amplitude & $\mathrm{n}$ & $\mathrm{m} \pm \mathrm{dp}$ & amplitude \\
\hline Idade (anos) & 111 & $13,6 \pm 2,4$ & $7-18$ & 95 & $13,3 \pm 2,5$ & $8-18$ \\
\hline IMC $\left(\mathrm{kg} / \mathrm{m}^{2}\right)$ & 111 & $21,8 \pm 2,7$ & $13,9-27,5$ & 95 & $21 \pm 2,1$ & $13,7-25,1$ \\
\hline
\end{tabular}

IMC: índice de massa corporal; m: média; dp: desvio-padrão.

Tabela 2. Valores medianos e de amplitude interquartílica dos níveis de AF e CS por geração e sexo.

\begin{tabular}{|c|c|c|c|c|c|c|c|}
\hline \multirow{2}{*}{\multicolumn{2}{|c|}{ Variáveis }} & \multicolumn{2}{|c|}{ 1.․ㅡ Geração } & \multicolumn{2}{|c|}{ 2. Geração } & \multicolumn{2}{|c|}{ 3. Geração } \\
\hline & & Avô & Avó & Pai & Mãe & Filho/Neto & Filha/Neta \\
\hline & & \multicolumn{2}{|c|}{ Me (IQR) } & \multicolumn{2}{|c|}{ Me (IQR) } & \multicolumn{2}{|c|}{ Me (IQR) } \\
\hline \multicolumn{2}{|c|}{ AFV (mets/sem) } & $0(0)$ & $0(0)$ & $0(320)$ & $0(160)$ & $280(960)$ & $80(640)$ \\
\hline \multicolumn{2}{|c|}{ AFM (mets/sem) } & $0(80)$ & $0(0)$ & $140(400)$ & $160(360)$ & $280(620)$ & $240(480)$ \\
\hline \multicolumn{2}{|c|}{ Caminhada (mets/sem) } & $330(297)$ & $247,5(256)$ & $743(660)$ & $660(561)$ & 718 (1068) & 743 (990) \\
\hline & $\mathrm{T}_{\mathrm{S}}(\mathrm{min} / \mathrm{dia})$ & $420(180)$ & $420(120)$ & $300(120)$ & $300(120)$ & $360(120)$ & $360(120)$ \\
\hline & $\mathrm{T}_{\mathrm{TV}}(\mathrm{min} / \mathrm{dia})$ & 155 (138) & $180(120)$ & $80(75)$ & $80(60)$ & $92,5(80)$ & $105(60)$ \\
\hline \multicolumn{2}{|c|}{ AFT (mets/sem) } & $413(291)$ & 264 (346) & 1058 (923) & 1015 (913) & $1722,25(1571)$ & 1545 \\
\hline
\end{tabular}

Me: mediana; IQR: amplitude interquartílica; AFV: atividade física vigorosa; AFM: atividade física moderada; CS: comportamento sedentário; $\mathrm{T}_{\mathrm{S}}$ : tempo sentado; $\mathrm{T}_{\mathrm{TV}}$ : tempo a assistir televisão; $\mathrm{AFT}$ : atividade física total; mets/sem: mets por semana; min/dia: minutos por dia.

A Figura 1 representa, graficamente, os valores do fenótipo AFT, agrupados em função da mediana dos valores de cada família. Desse modo, é possível visualizar a agregação familiar (AgF) nesse fenótipo por meio da variação substancial representada

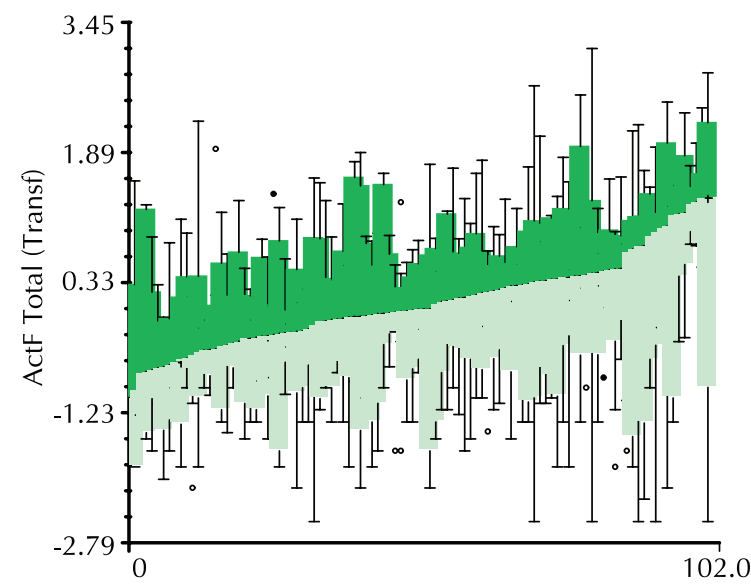

Figura 1. Distribuição dos diagramas de extremos e quartis das famílias no índice de AFT (100 famílias). entre as famílias. Para o fenótipo do CS, $\mathrm{T}_{\mathrm{TV}}$, a variabilidade apresentada (Figura 2) sugere um incremento menor da mediana entre as famílias, ou seja, $\mathrm{AgF}$ de menor magnitude do que o fenótipo da AF, supracitados.

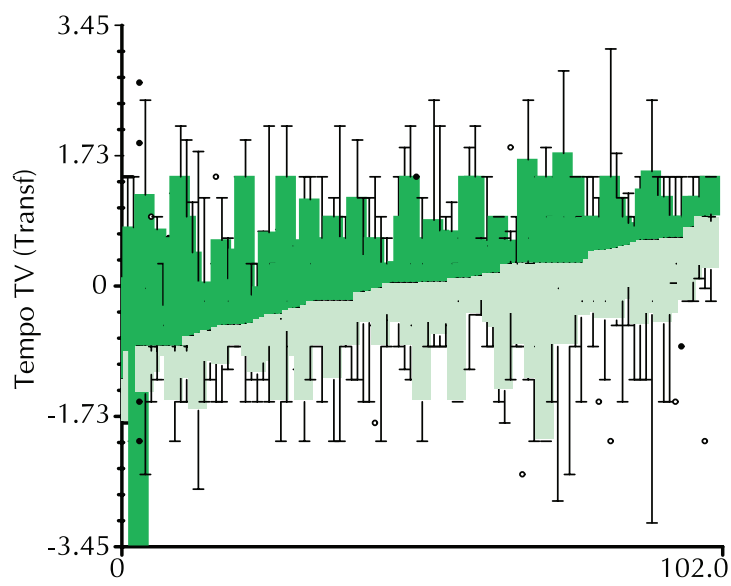

Figura 2. Distribuição dos diagramas de extremos e quartis das famílias no índice de CS (TTV) - (100 famílias). 
Tabela 3. Teste dos modelos para os fenótipos da AF e CS, valores de qui-quadrado obtidos pelas hipóteses testadas e estimativas de $\mathrm{h}^{2}$ (erro-padrão) ajustadas para covariáveis.

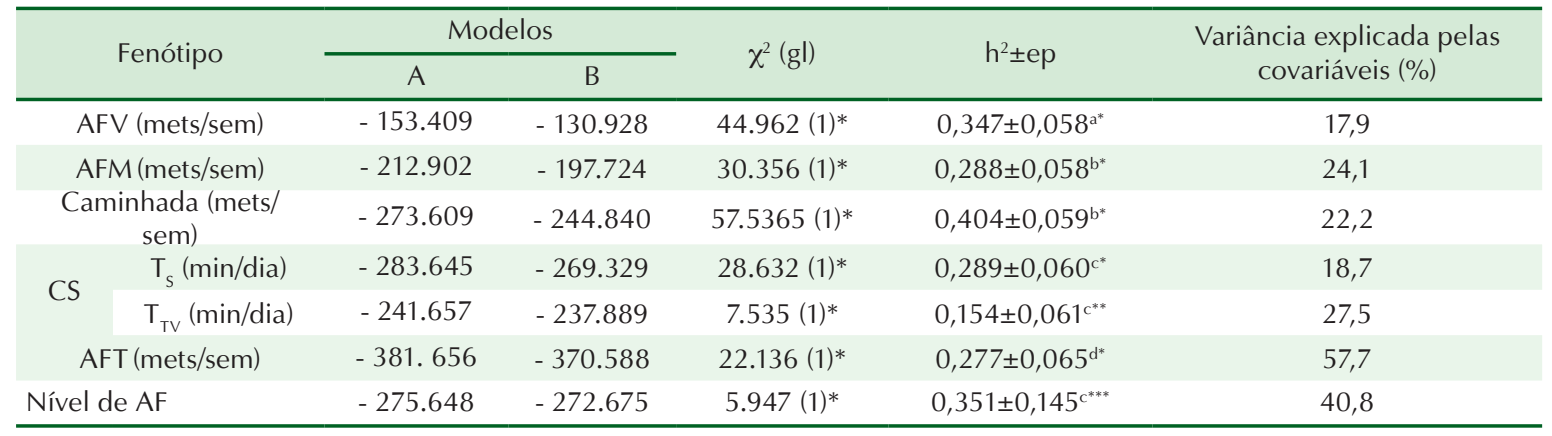

A: Valor do loglikelihood do Modelo esporádico (variância residual); B: Valor do loglikelihood do Modelo poligênico (efeitos aditivos genéticos); $\chi^{2}$ : qui-quadrado; gl: grau de liberdade; $h^{2}$ : estimativa de heritabilidade; ep: erro padrão. As demais abreviaturas encontram-se descritas na Tabela 2 . Covariáveis ajustadas: ${ }^{a}$ sexo; ${ }^{b}$ idade, idade ${ }^{2}$ e sexo*idade; ${ }^{c}$ idade e idade ${ }^{2}$; ${ }^{d}$ sexo, idade, idade ${ }^{2}{ }^{*} \mathrm{p}<0.0001 ;{ }^{* *} \mathrm{p} \leq 0,003$ * $^{* *} \mathrm{p} \leq 0,007$.

Quanto às estimativas de $\mathrm{h}^{2}$, variam entre baixa a moderada $(0,154-0,404)$, sendo todas estatisticamente significativas. $\mathrm{O}$ menor resultado foi para o fenótipo $T_{\mathrm{TV}}$ e o maior para caminhada. A fração da componente genética na determinação de ser pouco ativo ou apresentar atividade moderada a extenuante foi de 35\%. Os efeitos combinados da variância total atribuídos às diferentes covariáveis variam entre 18 e 58\%, sendo que o IMC não mostrou significância estatística. $\mathrm{O}$ ambiente partilhado não apresentou resultados significativos na influência dos níveis de AF e CS.

\section{DISCUSSÃO}

Tal como foi referido anteriormente, os própositos deste estudo foram verificar a presença indireta de transmissão vertical dos fatores genéticos e estimar a magnitude desses fatores, bem como da influência ambiental, na variabilidade dos níveis de AF e do CS. Contudo, é importante que sejam mencionados e comparados os valores de AF dos indivíduos das diferentes gerações.

Em geral, os valores medianos apresentados corroboram informação prévia ${ }^{6,24,}$ sendo consistentes com a evidência de redução dos níveis de AF ao longo da idade; mostram, também, uma certa instabilidade intergeracional no tempo dispendido em CS. Os indivíduos com mais de 50 anos tendem a apresentar valores de gasto energético com AFV próximos de zero, face a uma redução substancial da sua participação em atividades de maior intensidade. Quanto ao tempo dispendido em CS ( $\mathrm{T}_{\mathrm{S}}$ e $\left.\mathrm{T}_{\mathrm{TV}}\right)$, foi observado que, entre jovens e adultos idosos (primeira e terceira gerações), os valores são mais elevados comparados aos adultos jovens (segunda geração). Fato que pode ser devido às ocupações diárias de cada estrato etário.
Dos fenótipos abordados pelo presente estudo, a AFT apresenta mais elementos de comparação, sendo reportada com mais frequência em pesquisas anteriores $^{10,13,15-17}$. Ao ser avaliada por meio de questionários autoadministrados, as estimativas de $\mathrm{h}^{2}$ correspondem a $19 \%^{15}, 23 \%^{10}$ e $29 \%^{13}$, corroborando o valor de $28 \%$, sugerido para esta amostra. Os resultados com base na acelerometria indicam maior contribuição da componente genética nesse fenótipo. Por exemplo, Butte et al. ${ }^{17}$ e Cai et al. ${ }^{16}$, em um estudo com 1030 crianças de 319 famílias nucleares, participantes do estudo Viva La Familia, estimaram $\mathrm{h}^{2}$ para AFT de 55\% e 57\%, respectivamente.

Para os fenótipos AFV e AFM as estimativas de $h^{2}$ encontradas no presente estudo foram de 35 e 29\%. Simonen et al. ${ }^{15}$, no Québec Family Study, referem-se a esses fenótipos como AF moderada-a-extenuante, sendo avaliados, também, por meio de um questionário. Nesse estudo, os dados das 200 famílias indicam que a influência genética é responsável por explicar 16\% da variação total do fenótipo em causa. Em contrapartida, estudos baseados na acelerometria ${ }^{16-17}$ apresentam $\mathrm{h}^{2}$ de 18 e $32 \%$ para AFV, enquanto para AFM a h ${ }^{2}$ estimada é de 49 e $50 \%$. Não obstante a variabilidade de estimativas reportadas, os resultados encontrados evidenciam que os fatores genéticos exercem importante e significativa influência nesses fenótipos.

No presente estudo, o valor mais elevado de $h^{2}$ foi para a caminhada, $40 \%$ da variação fenotípica total. Dos estudos com delineamento familiar disponíveis, não foi encontrada referência a esse fenótipo, tendo em vista que não é avaliado pelos instrumentos utilizados em tais pesquisas. No entanto, o estudo gemelar longitudinal conduzido por Ortega et al. ${ }^{25}$, com uma amostra de 63 irmãs-gêmeas monozigóticas e 67 dizigóticas, participantes do Finnish Twin Cohort 
Study, salientou a influência dos fatores genéticos e ambientais em dois fenótipos da caminhada (velocidade e resistência) ao longo do tempo. Os resultados fornecem indícios de que a componente genética pode explicar entre 56 a $60 \%$ da variação total da velocidade e 40 a $60 \%$ da resistência.

As estimativas de $\mathrm{h}^{2}$ para os fenótipos que expressam o $C S$ foram de $15 \%$ para $T_{T V}$ e $29 \%$ para $T_{S}$. Das investigações analisadas, duas referem-se ao CS, salientando valores moderados de $h^{2}, 59$ e $60 \%{ }^{16-17}$, consideravelmente superiores a esta pesquisa. Ressalta-se que estão baseados em dados com acelerômetros, avaliando as atividades relacionadas a esse traço de forma conjunta. Simonen et al. ${ }^{15}$ sugerem que a contribuição relativa dos fatores genéticos ao fenótipo inatividade física (IF) corresponde a 25\%, sendo mais próximo aos providenciados pela presente pesquisa. Evidências da influência genética nesse fenótipo avançaram para o território da Genética Molecular, com a identificação de região cromossômica que possa albergar genes candidatos e/ou mutações responsáveis pela variação na IF. Com um estudo de genome-wide linkage scan para os níveis de AF, Simonen et al. ${ }^{26}$ encontraram linkage promissor no cromossomo $2 \mathrm{p} 22$ 16, detectando que a determinação de ser sedentário ou fisicamente ativo tem significativa contribuição genética e que há sentido em avançar para estudos de associação com genes candidatos.

É importante salientar que há estudos, baseados em pedigrees extensos, em que o efeito do ambiente partilhado não apresentou valores significativos na influência dos fenótipos avaliados. Por exemplo, Mitchell et al. ${ }^{12}$ estimaram a proporção da variação na AF como um efeito do ambiente comum e não encontraram valor significativo. Também Choh et al. ${ }^{13}$ observaram tal efeito apenas para AF de tempo-livre, não sendo significativo para a AFT, AF desportiva e AF no trabalho/escola. Em estudo com famílias nucleares, Pérusse et al. ${ }^{11}$ reportaram influência dos fatores ambientais comuns somente para participação em atividade desportiva e não para os níveis habituais de AF. Não obstante a discrepância nos resultados mencionados, a estimação do efeito do ambiente partilhado é ainda problemática em estudos com famílias cujos filhos não sejam gêmeos. As inconsistências reportadas podem ser atribuídas a componentes não mensuráveis do ambiente partilhado, como por exemplo, atitudes em relação à AF, características próprias do contexto em que a família está envolvida e aspectos da vizinhançç ${ }^{13}$.

Os efeitos ambientais (não transmitidos) contribuem entre 60 e $85 \%$ na variabilidade dos fenótipos avaliados nesta pesquisa, corroborando resultados previamente relatados ${ }^{11-13}$, cujo cálculo controla os efeitos do ambiente partilhado. Pérusse et al. ${ }^{11}$ ressaltam que $71 \%$ da variação nos níveis habituais de AF devem-se a fatores do ambiente. Choh et al. ${ }^{13}$ relatam valores entre $71 \%$ e $87 \%$ nos diferentes fenótipos da AF e Mitchell et al..$^{12}$ sugerem um valor pouco superior de $91 \%$. Todas as estimativas convergem ao fato do ambiente apresentar maior contribuição na variância total dos diferentes fenótipos relacionados à AF.

$O$ presente estudo tem pontos fortes, sobretudo, importantes por se situarem no domínio da pesquisa em Epidemiologia Genética aplicada à AF. Ressalta-se a magnitude amostral e etária dos participantes, bem como a presença de três gerações, constituindo um design com pedigrees extensos, pouco reportado na literatura. Destaca-se, também, a estimação do efeito do ambiente partilhado com base numa estrutura matricial familiar nos diferentes fenótipos da AF e CS.

Entre as limitações do estudo, destacam-se: (1) o uso de questionário autoadministrado para mensurar os níveis de AF e CS. Contudo, em pesquisas de grande dimensão em termos amostrais, é mais ou menos inevitável o recurso a questionários; (2) a estimativa do efeito do ambiente partilhado é sempre um problema porque se trata de uma variável latente. A necessidade de incorporar medidas mais objetivas de diferentes aspectos das vivências no seio das famílias pode ajudar a estimar de modo mais preciso a magnitude deste efeito.

Em suma, os resultados do presente estudo revelam presença significativa da transmissão dos fatores genéticos nos níveis de AF e CS. As estimativas de $\mathrm{h}^{2}$ explicam $28-40 \%$ da variação total nos níveis de AF e 15-29\% no CS. O efeito do ambiente partilhado não foi significativo para esta amostra, enquanto os efeitos ambientais não transmitidos apresentaram maior contribuição na variabilidade dos fenótipos avaliados. Contudo, é inequívoca a sugestão da presença de efeitos genéticos aditivos de magnitude baixa a moderada na variação total dos níveis de AF e CS. Portanto, os dados desta pesquisa contribuem para o desenvolvimento de estudos em Genética Molecular, ainda escassos, constituindo um suporte relevante a investigações e intervenções futuras.

\section{Agradecimentos}

Trabalho financiado pela Fundação para a Ciência e Tecnologia (FCT) de Portugal com a referência PTDC/DES/67569/2006. 


\section{REFERÊNCIAS BIBLIOGRÁFICA}

1. Akbartabartoori M, Lean ME, Hankey CR. The associations between current recommendation for physical activity and cardiovascular risks associated with obesity. Eur J Clin Nutr 2008;62(1):1-9.

2. Warburton DE, Nicol CW, Bredin SS. Health benefits of physical activity: the evidence. CMAJ 2006;174(6):801-9.

3. Pate RR, Pratt M, Blair SN, Haskell WL, Macera CA, Bouchard C, et al. Physical activity and public health. A recommendation from the Centers for Disease Control and Prevention and the American College of Sports Medicine. JAMA 1995;273(5):402-7.

4. Kesaniemi YK, Danforth E, Jr., Jensen MD, Kopelman PG, Lefebvre P, Reeder BA. Dose-response issues concerning physical activity and health: an evidence-based symposium. Med Sci Sports Exerc 2001;33(6 Suppl):S351-8.

5. Haskell WL, Lee IM, Pate RR, Powell KE, Blair SN, Franklin BA, et al. Physical activity and public health - Updated recommendation for adults from the American college of sports medicine and the American heart association. Circulation 2007;116(9):1081-93.

6. Pan SY, Cameron C, Desmeules M, Morrison H, Craig CL, Jiang X. Individual, social, environmental, and physical environmental correlates with physical activity among Canadians: a cross-sectional study. BMC Public Health 2009;9:21.

7. Trost SG, Owen N, Bauman AE, Sallis JF, Brown W. Correlates of adults' participation in physical activity: review and update. Med Sci Sports Exerc 2002;34(12):1996-2001.

8. Ferreira I, van der Horst K, Wendel-Vos W, Kremers S, van Lenthe FJ, Brug J. Environmental correlates of physical activity in youth - a review and update. Obes Rev 2007;8(2):129-54.

9. Sallis JF, Prochaska JJ, Taylor WC. A review of correlates of physical activity of children and adolescents. Med Sci Sports Exerc 2000;32(5):963-75.

10. Seabra AF, Mendonca DM, Goring HHH, Thomis MA, Maia JA. Genetic and environmental factors in familial clustering in physical activity. Eur J Epidemiol 2008;23(3):205-11.

11. Perusse L, Tremblay A, Leblanc C, Bouchard C. Genetic and environmental influences on level of habitual physical activity and exercise participation. Am J Epidemiol 1989;129(5):1012-22.

12. Mitchell BD, Rainwater DL, Hsueh WC, Kennedy AJ, Stern MP, Maccluer JW. Familial aggregation of nutrient intake and physical activity: Results from the San Antonio Family Heart Study. Ann Epidemiol 2003;13(2):128-35.

13. Choh AC, Demerath EW, Lee M, Williams KD, Towne B, Siervogel RM, et al. Genetic analysis of self-reported physical activity and adiposity: the Southwest Ohio Family Study. Public Health Nutr 2008;12(8):1052-60.
14. Campos MAOA, Maia JA. Combata a síndrome metabólica. Cuide da sua família e faça actividade física. Porto: FADEUP DRD-RAA; 2007.

15. Simonen RL, Perusse L, Rankinen T, Rice T, Rao DC, Bouchard C. Familial aggregation of physical activity levels in the Quebec family study. Med Sci Sport Exer 2002;34(7):1137-42.

16. Cai G, Cole SA, Butte N, Bacino C, Diego V, Tan K, et al. A quantitative trait locus on chromosome $18 \mathrm{q}$ for physical activity and dietary intake in Hispanic children. Obesity (Silver Spring) 2006;14(9):1596-604.

17. Butte NF, Cai GW, Cole SA, Comuzzie AG. Viva la Familia Study: genetic and environmental contributions to childhood obesity and its comorbidities in the Hispanic population. Am J Clin Nutr 2006;84(3):646-54.

18. Rankinen T, Bouchard C. Individual Responses to Physical Activity. The Role of Genetics. In: Lee IM, Blair S, Manson J, Paffenbarger Jr RS, organizadores. Epidemiologic Methods in Physical Activity Studies. New York: Oxford; 2009. p.77-99

19. Rice TK, Borecki IB. Familial Resemblance and Heritability. In: Rao DC, Province MA, editors. Genetics Dissection of Complex. San Diego, 2001. p.35-44.

20. Craig CL, Marshall AL, Sjostrom M, Bauman AE, Booth ML, Ainsworth BE, et al. International physical activity questionnaire: 12 -country reliability and validity. Med Sci Sports Exerc 2003;35(8):1381-95.

21. Bauman A, Bull F, Chey T, Craig CL, Ainsworth BE, Sallis JF, et al. The International Prevalence Study on Physical Activity: results from 20 countries. Int J Behav Nutr Phys Act 2009;6(1):21.

22. IPAQ. Guidelines for Data Processing and Analysis of the International Physical Activity Questionnaire. 2005; Available from: <http://www.ipaq.ki.se/scoring.pdf $>$.

23. Lohman TG, Roche AF, Martorell R. Antropometric standardization reference manual. Champaign: Human Kinetics; 1988.

24. Hagströmer M, Bergman P, Baunan A, Sjöstrïn M. The international prevalence study (IPS): health-enhancing physical activity in Sweden. J Public Health 2006;14:301-8.

25. Ortega-Alonso A, Sipila S, Kujala UM, Kaprio J, Rantanen T. Longitudinal changes in genetic and environmental influences on older women's walking ability. Scand J Med Sci Sports 2009;19(5):669-77.

26. Simonen RL, Rankinen T, Perusse L, Rice T, Rao DC, Chagnon Y, et al. Genome-wide linkage scan for physical activity levels in the Quebec Family study. Med Sci Sports Exerc 2003;35(8):1355-9.
Endereço para correspondência
José António Ribeiro Maia
Rua Dr. Plácido Costa, 91
4200.450 Porto, Portugal
E-mail: jmaia@ fade.up.pt 